\title{
Calidad metodológica de los ensayos clínicos de cicatrices queloides
}

\author{
Methodological quality of clinical trials of keloid scars
}

Martha A. Morales-Sánchez*, Andrea De Villafranca-Dugelby, Fermín Jurado-Santa Cruz y

María L. Peralta-Pedrero

Unidad de Investigación del Centro Dermatológico Dr. Ladislao de la Pascua, Servicios de Salud Pública de la Ciudad de México, Ciudad de México, México

\section{Resumen}

Antecedentes: Existe heterogeneidad en el diseño de los ensayos clínicos (EC) para el tratamiento de cicatrices queloides que compromete la validez de sus resultados. Objetivo: Evaluar la calidad metodológica de los EC publicados sobre cicatrices queloides, principalmente las medidas de eficacia utilizadas. Método: Se analizaron los EC publicados sobre tratamientos para cicatrices queloides y se evaluó su calidad metodológica siguiendo los lineamientos CONSORT y el riesgo de sesgo según la herramienta de Cochrane. Se identificaron todas las medidas de eficacia utilizadas en los EC. Resultados: Se incluyeron 52 artículos, de los cuales solo en el 3.84\% se mencionan los cambios importantes en la metodología después de iniciar el reclutamiento de los pacientes. El 59.6\% de los EC fueron evaluados con alto riesgo de sesgo de realización por errores en el cegamiento del equipo de investigación. La variable de respuesta más frecuente fue la altura o grosor de las cicatrices. Conclusiones: Se recomienda que en los EC de cicatrices queloides se excluya a los participantes con cicatrices hipertróficas, y que la variable de resultado principal sea la altura de la cicatriz. También deben medirse el dolor y la calidad de vida de los pacientes.

Palabras clave: Cicatrices. Ensayos clínicos. Queloides. Riesgo de sesgo.

\begin{abstract}
Background: There is heterogeneity in the design of clinical trials (CT) for the treatment of keloid scars that compromises the validity of their results. Objective: To assess the methodological quality of the CT published on keloid scars, mainly the outcomes used in them. Method: Articles of CT for keloid scars were analyzed, their methodological quality was evaluated following the CONSORT guidelines and the risk of bias based on the Cochrane tool. All the clinical outcomes measured in CT were identified. Results: Fifty-two full-text articles were evaluated, of which, only 3.84\% of the CT mentioned important changes in the methodology after starting patient recruitment. Fifty-nine percent of the CT were assessed as high risk of performance bias due to mistakes in blinding participants and personnel. The most frequent outcome was the height or thickness of the keloids. Conclusions: We recommend that participants with hypertrophic scars should be excluded from keloids' clinical trials, and that the main outcome must be the scar height and flattening. The pain and quality of life of patients should also be measured.
\end{abstract}

Key words: Scars. Clinical trials. Keloids. Risk of bias.

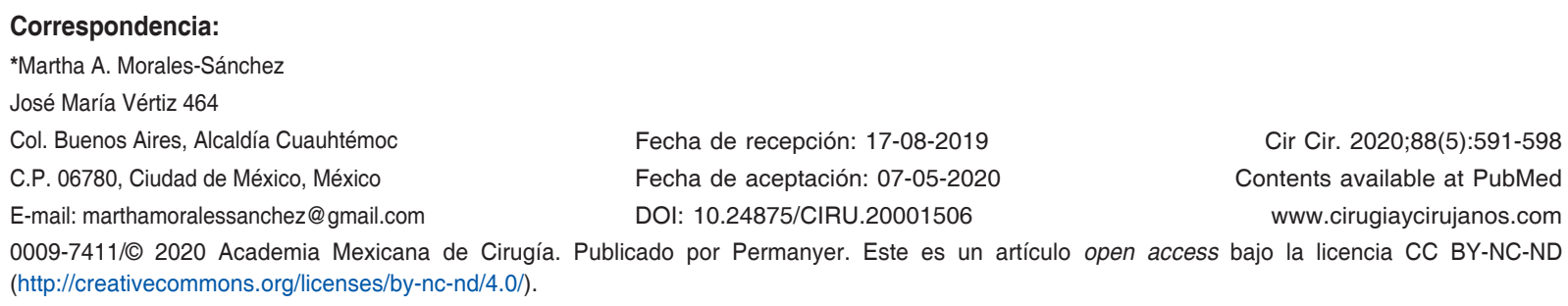

Cir Cir. 2020;88(5):591-598 Contents available at PubMed www.cirugiaycirujanos.com 0009-7411/@ 2020 Academia Mexicana de Cirugía. Publicado por Permanyer. Este es un artículo open access bajo la licencia CC BY-NC-ND (http://creativecommons.org/licenses/by-nc-nd/4.0/). 


\section{Introducción}

Una cicatriz es una respuesta fisiológica a una solución de continuidad de cualquier tejido. En la piel, las cicatrices se clasifican en normotróficas, atróficas, hipertróficas y queloides ${ }^{1,2}$. Las cicatrices queloides son una alteración en la reparación de las heridas en individuos genéticamente susceptibles, caracterizada por la síntesis exagerada de tejido conectivo ${ }^{3}$. El diagnóstico de las cicatrices queloides es fácil para un médico; sin embargo, el reto es encontrar un tratamiento efectivo que disminuya también el riesgo de recurrencias. Los ensayos clínicos (EC) aleatorizados son el método de referencia para medir la eficacia y la seguridad de un tratamiento, y parten de evaluar el cambio en las cicatrices queloides después de cualquier intervención farmacológica o quirúrgica.

Para evaluar el cambio en las cicatrices queloides se han diseñado diversas escalas, siendo la más utilizada la Escala de Vancouver. Sin embargo, en las últimas tres décadas se han creado otras escalas para medir con detalle la pigmentación, la vascularización, el grosor y la apariencia de las cicatrices, como las escalas POSAS (Patient and Observer Scar Assessment Scale), MAPS (Assessment of Scars and Photographs) y MSS (Manchester Scar Scale), entre otras. Adicionalmente, se ha utilizado la escala visual análoga para medir el dolor y el prurito desencadenados por las cicatrices queloides. La existencia de varias formas de medir los cambios en las cicatrices queloides ha ocasionado que los EC sobre ellas sean poco comparables tan solo en medir la eficacia de las intervenciones; además, la evaluación de las cicatrices mediante una escala o un índice clínico es dependiente del operador. Lo anterior se suma a lo encontrado en los tres metaanálisis más recientes sobre intervenciones para cicatrices queloides, en los que los autores encontraron EC con tamaños de muestra pequeños, heterogeneidad en la evaluación de la eficacia y falta de información sobre el enmascaramiento de las intervenciones y la persona del equipo de investigación que realiza la medición de las cicatrices ${ }^{4-6}$. Solo en el metaanálisis de $\mathrm{Bi}$, et al. ${ }^{6}$ se encontró una alta comparabilidad de los resultados, debido a que la mayoría de los 14 estudios incluidos los habían realizado integrantes de un mismo equipo de investigación.

La calidad de un EC aleatorizado se basa en su diseño, la medición de la variable de resultado (la forma de medir el efecto del tratamiento) y el análisis estadístico de sus resultados, lo cual debe reflejarse en el artículo publicado. Un artículo que no incluya la información suficiente para evaluar su calidad no permite al médico emitir un juicio sobre su validez, tanto interna como externa, y hace que se cuestione su aporte científico, no se confíe en sus resultados y probablemente no se utilice en la práctica clínica cotidiana ${ }^{1,7}$. Por ello, actualmente la Colaboración Cochrane y la iniciativa CONSORT (Consolidated Standards of Reporting Trials) han impulsado la evaluación crítica de la calidad metodológica de los EC aleatorizados para garantizar que solo las intervenciones que sean efectivas lleguen a los pacientes 4 .

En cicatrices queloides, al no existir un tratamiento altamente efectivo, aunado al costo elevado de los disponibles, es necesario analizar si la evidencia obtenida en los EC publicados es real o está sobreestimada por errores metodológicos. Por lo anterior, el objetivo de esta revisión sistemática fue evaluar las características metodológicas de los EC publicados sobre cicatrices queloides, principalmente las medidas de eficacia utilizadas en ellos, y determinar su riesgo de sesgo, con la finalidad de concluir con recomendaciones para los investigadores clínicos involucrados en el diseño y la ejecución de proyectos de investigación sobre el tema.

\section{Método}

Se realizó una revisión sistemática sobre la metodología de los EC en cicatrices queloides centrada en las formas de medir la eficacia o los cambios en las cicatrices, cuyo protocolo fue registrado en 2018 en la plataforma PROSPERO con el número de registro CRD42018107933'.

\section{Estrategia de búsqueda}

Se realizó una búsqueda en las bases de datos MEDLINE y Embase vía PubMed y OVID SP, y en SciELO (Scientific Electronic Library Online), desde 1970 hasta agosto de 2018. Se utilizaron como palabras clave "keloid" y "scar", limitando por diseño de estudio para "clinical trial", sin restricción de idioma ni grupo de edad.

\section{Selección de los estudios}

Dos autores de forma independiente (M.L. Peralta-Pedrero y M.A. Morales-Sánchez) revisaron la búsqueda bibliográfica para identificar, a través de los títulos y los resúmenes de los artículos, cuáles 
cumplían los criterios de selección. Posteriormente se obtuvieron los artículos seleccionados a texto completo para verificar los criterios de selección. Las discrepancias sobre la inclusión de algún estudio se resolvían por consenso entre ambos autores.

\section{Criterios de selección}

Se incluyeron EC que hubieran reclutado participantes con cicatrices queloides. Se excluyeron los estudios cuasiexperimentales y aquellos que hubieran reclutado principalmente participantes con cicatrices de acné (en rollo, caja y picahielo) y por quemaduras.

\section{Extracción de datos}

Dos autores (M.A. Morales-Sánchez y A. De Villafranca-Dugelby) evaluaron el texto completo de los estudios incluidos para recuperar las características de los ensayos, la muestra reclutada, la variable para medir la eficacia y los resultados principales, y para analizar el riesgo de sesgo.

\section{Evaluación de la metodología y del riesgo de sesgo}

Para evaluar la metodología se utilizaron como guía los estándares del grupo CONSORT, y para el riesgo de sesgo se usó la herramienta diseñada por la Colaboración Cochrane, que incluye los dominios de generación de la secuencia de aleatorización, enmascaramiento, datos incompletos y reporte selectivo, entre otros ${ }^{9,10}$. La propuesta CONSORT surgió en 1996 con el fin de estandarizar diferentes criterios para reportar los EC aleatorizados y para facilitar su lectura crítica y su interpretación. En 2001 se revisaron los lineamientos y en 2010 se publicó una tercera y última versión. Se conforma por 25 ítems agrupados en seis dominios: título y resumen, introducción, metodología, resultados, discusión y otra información, como el registro del protocolo original y el financiamiento con el cual se llevó a cabo el estudio. Dos autores de forma independiente (M.A. Morales-Sánchez y A. De Villafranca-Dugelby) realizaron la evaluación y otros dos (M.L. Peralta-Pedrero y F. Jurado-Santa Cruz) la revisaron para llegar a un consenso.

\section{Análisis estadístico}

Al ser una revisión sistemática solo se reportan proporciones de las características del estudio y de los dominios del riesgo de sesgo. No se realizó una síntesis cuantitativa ni un metaanálisis de los EC analizados.

\section{Resultados}

Se encontraron 1,446 artículos, de los cuales se evaluaron los 52 que cumplían con los criterios de selección. En el Anexo 1 se desglosan los artículos de los EC incluidos por autor y referencia bibliográfica. El diagrama de flujo de la inclusión de estudios se muestra en la figura 1.

\section{Características del diseño de los ensayos clínicos}

Del total de los EC, el $80.8 \%(n=42)$ tuvieron un diseño de grupos paralelos y el $19.2 \%(n=10)$ cruzado, en los que cada participante fue su propio control. La mayoría de los EC tuvieron dos grupos de intervención; solo uno comparó cuatro intervenciones de forma simultánea. La asignación a las intervenciones únicamente fue aleatoria en el $63.5 \%(n=33)$ de los EC; en un $17.3 \%(n=9)$ de los artículos no se menciona como se realizó dicha asignación. El cegamiento de las intervenciones se realizó en el $42.3 \%$ $(n=22)$ de los EC; en el $40.4 \%(n=21)$ no se especifica, mientras que en el $17.3 \%(n=9)$ no fue posible por el tipo de intervención. De los 22 EC con cegamiento, la intervención se enmascaró para el evaluador de la medida de eficacia en el $68.2 \%(n=15)$, para el evaluador y el participante (doble ciego) en el $22.7 \%(n=5)$ y solo para el participante en el $9.1 \%$ $(n=2)$. El número de participantes reclutados por EC osciló entre 5 y 176 (mediana 31).

\section{Características de los participantes}

En el $50 \%(n=26)$ de los EC se reclutaron participantes con cicatrices queloides; en el resto se reclutaron también con cicatrices hipertróficas. En poco más de la mitad de los EC $(51.9 \%, n=27)$ los sujetos de estudio fueron adultos; el $32.7 \%(n=17)$ incluyeron niños y adultos, y el $5.8 \%(n=3)$ reclutaron solo población pediátrica. En el $9.6 \%(n=5)$ de los EC no se reportó la edad de los pacientes en los criterios de selección ni en la sección de resultados. La topografía de la cicatriz se reportó en el $69.2 \%$ de los EC $(n=36)$, principalmente en la cabeza, el tronco y las extremidades. El $50 \%$ de los EC $(n=26)$ reportó el origen de la cicatriz, siendo la mayoría por 

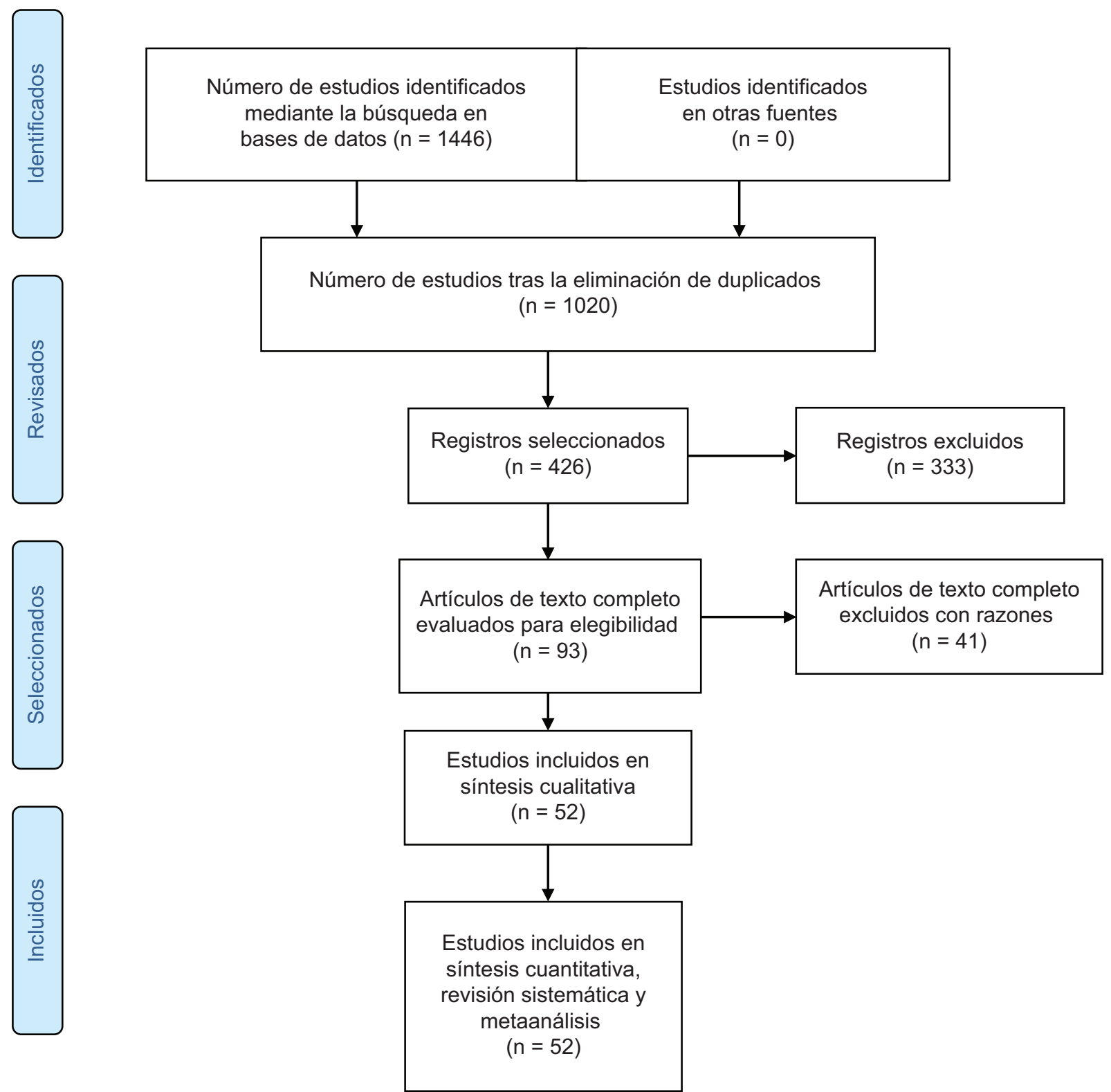

Figura 1. Diagrama de flujo PRISMA.

procedimientos quirúrgicos, quemaduras y acné, mientras que solo en el $42.3 \%(n=22)$ se reporta el tiempo de evolución, el cual osciló desde 1 día hasta 280 días.

\section{Intervenciones}

El tipo de intervención que se probó en los EC fue un tratamiento farmacológico en el $65.4 \%$, con administración tópica o intralesional, seguido por los parches de silicón en el $44.2 \%$, criocirugía en el $30.8 \%$, extirpación quirúrgica en el $27 \%$ y láser en el $19.2 \%$. Siete estudios tuvieron grupo control con placebo.

\section{Medición de la eficacia}

La medida de eficacia más utilizada fue la altura o grosor de la cicatriz, en el $75 \%$ de los estudios incluidos, seguida del uso de una escala o índice clínico en el $71.2 \%$, y de la elasticidad y el eritema o la vascularidad con el $55.8 \%$ cada una. Para evaluar la altura o grosor de la cicatriz, solo en 13 artículos se menciona que utilizaron una regla graduada. Los síntomas que se consideraron con mayor frecuencia como variables de respuesta fueron el prurito, en el $50 \%$ de los EC, y el dolor, en el $34.6 \%$. En el $21.2 \%$ $(n=11)$ de los EC se midió el dolor percibido por el 
Tabla 1. Medidas de eficacia de los ensayos clínicos sobre cicatrices queloides

\begin{tabular}{lc}
\hline Variable & \% ( $\mathbf{n} \mathbf{5 2})$ \\
\hline Altura de la cicatriz & $75(39)$ \\
Escala o índice clínico & $71.2(37)$ \\
Elasticidad & $55.8(29)$ \\
Eritema o vascularidad & $55.8(29)$ \\
Prurito & $50(26)$ \\
Pigmentación & $40.4(21)$ \\
Aplanamiento & $28.8(15)$ \\
Dolor & $34.6(18)$ \\
Área de la cicatriz & $26.9(14)$ \\
Irregularidad de la superficie & $13.5(7)$ \\
Volumen & $1.9(1)$ \\
Limitación de la movilidad & $1.9(1)$ \\
\hline
\end{tabular}

paciente al momento de la administración de las intervenciones. El aplanamiento o altura cero de las cicatrices solo se consideró como variable de respuesta o desenlace en el $28.8 \%$ de los EC. El resto de las medidas de eficacia incluidas en los EC se muestran en la tabla 1. Es importante mencionar que únicamente en el $25 \%$ de los EC $(n=13)$ se evaluó la recurrencia de la cicatriz queloide posterior a las intervenciones, y el tiempo al que se evaluó fue desde 6 meses posterior al inicio de la intervención hasta 5 años, con una mediana de tiempo de 12 meses. Los autores reportaron la presencia de eventos adversos en 28 artículos (53.8\%) y los más frecuentes fueron eritema, dolor, hipopigmentación, atrofia, dermatitis y telangiectasias.

En cuanto a las escalas clínicas utilizadas para medir la eficacia de las intervenciones, la escala Vancouver se aplicó en el 21.2\% $(n=11)$ de los EC, seguida de las escalas de mejoría subjetivas aplicadas por el investigador en el $19.2 \%(n=10)$, las escalas de mejoría subjetiva dirigidas a los pacientes en el $17.3 \%(n=9)$ y la escala POSAS en el $7.7 \%(n=4)$. En el $19.2 \%(n=10)$ de los EC se utilizaron escalas o índices diseñados específicamente para dichos estudios, todos enfocados a realizar una evaluación subjetiva de la apariencia de las cicatrices y de los síntomas.

Con respecto a la evaluación de la eficacia o la medición de la variable de desenlace, solo en el $25 \%$
Tabla 2. Evaluación metodológica según CONSORT

\begin{tabular}{|c|c|c|}
\hline Dominio & Ítem & $\%(n=52)$ \\
\hline \multirow[t]{15}{*}{ Métodos } & $\begin{array}{l}\text { Identificado como un ensayo aleatorizado } \\
\text { en el título }\end{array}$ & $13.4(7)$ \\
\hline & Resumen estructurado & $84.6(44)$ \\
\hline & Antecedentes científicos y justificación & $94.23(49)$ \\
\hline & Objetivos específicos o hipótesis & $90.38(47)$ \\
\hline & $\begin{array}{l}\text { Descripción del diseño del ensayo, } \\
\text { incluida la razón de asignación }\end{array}$ & $94.23(49)$ \\
\hline & $\begin{array}{l}\text { Cambios importantes en los métodos } \\
\text { después de iniciar el ensayo }\end{array}$ & $3.84(2)$ \\
\hline & Criterios de selección de los participantes & $92.3(48)$ \\
\hline & $\begin{array}{l}\text { Procedencia (centros e instituciones) en } \\
\text { que se registraron los datos }\end{array}$ & $65.3(34)$ \\
\hline & Descripción detallada de la intervención & $98(51)$ \\
\hline & $\begin{array}{l}\text { Especificación a priori de las variables } \\
\text { respuesta }\end{array}$ & $94.23(49)$ \\
\hline & $\begin{array}{l}\text { Cualquier cambio en las variables } \\
\text { respuesta tras el inicio del ensayo }\end{array}$ & $9.6(5)$ \\
\hline & Cálculo del tamaño de muestra & $21.15(11)$ \\
\hline & $\begin{array}{l}\text { Si corresponde, explicar cualquier análisis } \\
\text { intermedio y las reglas de interrupción }\end{array}$ & $19.23(10)$ \\
\hline & $\begin{array}{l}\text { Método utilizado para generar la } \\
\text { secuencia de asignación aleatoria }\end{array}$ & $44.23(23)$ \\
\hline & $\begin{array}{l}\text { Tipo de aleatorización; detalles de } \\
\text { cualquier restricción }\end{array}$ & $42.3(22)$ \\
\hline
\end{tabular}

( $n=13)$ de los EC los autores mencionaron quién realizó tal medición. De los 13 EC, en nueve la evaluación fue realizada por un dermatólogo, mientras que en los restantes los evaluadores fueron cirujanos plásticos y patólogos (un estudio en el que los cambios histológicos fueron la variable de respuesta).

\section{Evaluación del contenido del manuscrito}

De acuerdo con los lineamientos CONSORT, en el $100 \%$ de los EC los resultados fueron consistentes en beneficios y daños considerando las evidencias científicas relevantes, mientras que en el $98 \%(n=51)$ las intervenciones para cada grupo fueron detalladas, permitiendo su replicación. Por otro lado, el $3.84 \%$ de los EC mencionaron los cambios importantes en la metodología después de iniciar el reclutamiento de los pacientes, y solo en un estudio se encontró a texto completo el protocolo de investigación. Únicamente 
Generación de secuencia aleatoria (sesgo de selección) Ocultamiento de la asignación (sesgo de selección) Cegamiento de los participantes e investigadores (sesgo de realización) Cegamiento de la evaluación (sesgo de detección) Datos de resultados incompletos (sesgo de desgaste) Reporte selectivo (sesgo de notificación) Otros sesgos

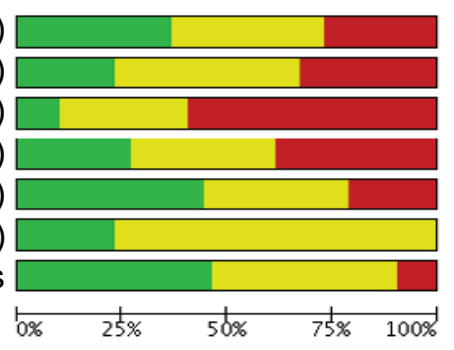

Figura 2. Gráfica de riesgo de sesgo.

de cinco EC se encontró su registro en alguna base de datos y se documentaron los cambios en las variables después de iniciado el reclutamiento de los participantes. El desglose del cumplimiento del contenido de los manuscritos se muestra en la tabla 2.

\section{Evaluación del riesgo de sesgo}

Del total de los EC, el 26.9\% ( $n=14)$ fueron clasificados con alto riesgo de sesgo de selección por no contar con un método adecuado para la generación de la secuencia de asignación a los grupos de intervención. El $32.7 \%(n=17)$ de los EC no utilizaron un buen método para ocultar la asignación a los grupos de intervención, independientemente de la generación de la secuencia aleatoria, por lo que también fueron clasificados con alto riesgo de sesgo de selección. Más de la mitad de los EC $(59.6 \%, n=31)$ fueron evaluados con alto riesgo de sesgo de realización por errores en el cegamiento, tanto de los participantes como de los investigadores, que pudieron influir en la variable de respuesta. Con relación al sesgo de detección, el $38.5 \%$ ( $n=20)$ de los estudios tuvieron alto riesgo porque los investigadores que evaluaron la respuesta a los tratamientos sabían la intervención asignada a los participantes que evaluaron, ya que no fue posible realizar un cegamiento adecuado. El $21.1 \%$ ( $n=11)$ de los EC no realizaron un análisis estadístico que incluyera a todos los pacientes reclutados y solo reportaron los resultados de los participantes que completaron las intervenciones; algunos no reportaron todas las variables que midieron según la metodología del artículo o las que debieron medir por las escalas o índices clínicos que utilizaron. Ningún EC fue clasificado con alto riesgo de sesgo de notificación, pero el $76 \%(n=40)$ fueron clasificados con riesgo poco claro de sesgo de notificación porque no se encontró el registro del protocolo y no midieron la eficacia de las intervenciones con los instrumentos adecuados. Cabe señalar que pocos EC fueron clasificados con bajo riesgo de sesgo en todos los dominios; de hecho, solo 5 (9.6\%) se clasificaron con bajo riesgo de sesgo de realización. El desglose de las evaluaciones del riesgo de sesgo se muestra en la figura 2. En el dominio de «otros sesgos» se evaluó que el $9.6 \%(n=5)$ de los EC tenían diferencias entre los grupos al inicio de las intervenciones o no se presentaban los datos para verificar la comparabilidad de los grupos de acuerdo con su perfil basal.

\section{Discusión}

En nuestra revisión sistemática encontramos que la medida de eficacia más utilizada en los EC para el tratamiento de las cicatrices queloides es la altura o el grosor de las cicatrices, ya que fue la variable de respuesta en el $75 \%$ de los EC, seguida del uso de alguna escala o índice clínico en el $71.2 \%$. Sin embargo, aunque la mayoría de los estudios utilizan una escala para evaluar las características de las cicatrices, la que más se emplea es la de Vancouver, en un $21.2 \%$ de los EC. Es decir, existe heterogeneidad en la forma de evaluar las cicatrices queloides cuando se utiliza una escala, a pesar de la existencia de revisiones sistemáticas sobre las propiedades de dichos instrumentos, que señalan que aquellas con el mejor proceso de validación son POSAS y PRISM (Patient-Reported Impact of Scars Measure), ya que ambas incluyen ítems que miden el impacto en los pacientes ${ }^{11}$. Sin embargo, estas escalas son largas, con poca correlación con medidas objetivas como altura, eritema, pigmentación y elasticidad, y PRISM, que es la más robusta, no mide la apariencia de las cicatrices ${ }^{12}$. Una de las ventajas de usar la escala de 
Vancouver es que está enfocada a evaluar características clínicas de las cicatrices, como altura, elasticidad, vascularidad y pigmentación, y además es de fácil aplicación para los investigadores clínicos a pesar de no tener una buena reproducibilidad interobservador, motivo por el cual en un EC debería ser siempre aplicada por un mismo evaluador para evitar errores de medición ${ }^{11}$. Por su parte, la escala POSAS no tiene sensibilidad al cambio en las características de las cicatrices durante un EC, a pesar de contar con muchos dominios para su evaluación ${ }^{12}$.

Metodológicamente, todos los EC deben incluir medidas de la eficacia de las intervenciones reportadas por los pacientes (patient-reported outcomes), dentro de las cuales en cicatrices queloides se midieron el prurito y el dolor en el $50 \%$ y el $34.6 \%$, respectivamente. En el $17.3 \%$ de los EC, el paciente evaluó su respuesta al tratamiento mediante una escala análoga visual de mejoría. Sin embargo, una revisión sistemática sobre instrumentos o escalas diseñados para medir cambios en las cicatrices queloides documentó que no existe correlación entre lo que reportan el paciente $y$ el investigador sobre las respuestas a los tratamientos $^{13}$. A pesar de la diferencia en dichas evaluaciones, en un EC sobre cicatrices queloides es indispensable medir la percepción del paciente y su calidad de vida, ya que es muy probable que el tratamiento no logre una mejoría clínicamente significativa, pero la modificación de la sintomatología podría ser un beneficio para el paciente. En este sentido, es importante señalar que en ninguno de los EC revisados se midió la calidad de vida.

Cuando evaluamos los EC de acuerdo con los lineamientos CONSORT, solo el $13.4 \%$ se identifican como EC aleatorizados en el título, en dos no se mencionan los cambios en la metodología posterior al reclutamiento de los pacientes y al inicio de las intervenciones, a pesar de que sí se realizaron dichos cambios, y solo en uno se encontró el protocolo completo. Lo anterior muestra la falta de rigor metodológico en la redacción de los EC y que en las revistas en que fueron publicados no se considera el apego a CONSORT como uno de los requisitos para la publicación. Actualmente, la mayoría de las revistas internacionales indexadas solicitan la lista de cotejo de CONSORT como requisito de envío para los manuscritos que reportan EC. Es interesante notar que solo la mitad de los artículos revisados (51.9\%) fueron publicados en revistas de dermatología; el resto aparecieron en revistas de cirugía y medicina en general.
Por otra parte, cuando evaluamos el riesgo de sesgo encontramos fallas importantes que afectan directamente los resultados de los EC, poniendo en duda su validez tanto interna como externa. El error más frecuente identificado fue la falla en el cegamiento del equipo de investigación y de los participantes a las intervenciones, lo cual impacta en los resultados cuando el evaluador de la respuesta al tratamiento o variable de resultado es el mismo médico que aplica el tratamiento y el paciente es conocedor de la terapia que recibe $e^{4,5}$. Además, la mayoría de las escalas utilizadas, como Vancouver y POSAS, son dependientes del observador y con concordancias intraobservador e interobservador, y por tanto el hecho de que los autores no reporten quién midió la intervención puede ser un factor para cuestionar los resultados.

Finalmente, el $26.9 \%$ de los EC tuvieron un alto riesgo de sesgo de selección por fallas en la generación de la secuencia de asignación a las intervenciones, lo cual compromete la calidad de los resultados de cualquier EC y en otras áreas de la medicina es raro que ocurra. Además, en la mayoría de los manuscritos no se reportó información esencial para poder evaluar el riesgo de sesgo de los EC.

Como recomendaciones derivadas de esta revisión sistemática proponemos como regla a seguir, en los EC sobre cicatrices queloides, el excluir del reclutamiento a pacientes con cicatrices hipertróficas, ya que en la mitad de los EC analizados se incluyeron ambas poblaciones y no se analizaron de forma separada. Por su evolución natural y su fisiopatología, las cicatrices hipertróficas tienden a mejorar a lo largo del tiempo y responder fácilmente al tratamiento de compresión, con parche de silicón y otros, al contrario que las cicatrices queloides, lo cual debe controlarse desde el diseño de los EC para evitar la confusión en el efecto del tratamiento ${ }^{14}$. La variable de resultado principal debe ser la altura o el grosor de la cicatriz queloide, y hay que reportar el porcentaje de participantes que alcanzaron el aplanamiento de la cicatriz o altura cero, ya que este es el resultado clínicamente significativo tanto para el paciente como para el investigador. Como variables de respuesta secundarias deben incluirse la medición del dolor y del prurito con escalas análogas visuales, y la calidad de vida utilizando cuestionarios genéricos o con la escala PRISM, que mide dicho impacto en los pacientes. La recurrencia de las cicatrices queloides debe ser otra variable por medir, pero al menos a 12 meses de la intervención; por lo 
tanto, recomendamos que un EC sobre cicatrices cuente con al menos 12 semanas de tratamiento, idealmente 6 meses, y seguimiento a 1 año. Dada la dificultad para enmascarar intervenciones con vías de administración diferentes, que es la regla en los tratamientos para las cicatrices queloides, nuestra sugerencia es que el evaluador de la respuesta al tratamiento sea independiente y esté cegado a las intervenciones; es decir, que no sea el investigador principal, el cual suele atender a los pacientes y puede reconocer fácilmente el tipo de intervención que está recibiendo y verse influenciado. Todas estas recomendaciones van dirigidas a buscar un consenso sobre cuáles deberían ser las variables de resultado a medir en los EC de cicatrices queloides, como ya se ha hecho en otras patologías ${ }^{15}$.

Las limitaciones de esta revisión sistemática son las inherentes a la falta de información encontrada en los manuscritos, hecho que refuerza la hipótesis de que en los EC para cicatrices queloides debe realizarse un consenso de expertos para definir las variables de respuesta al tratamiento que es necesario medir, los procedimientos de los EC y el apego a los lineamientos internacionales sobre publicación de artículos científicos.

\section{Conclusión}

Esta revisión sistemática nos permitió conocer las fallas en el diseño y en el reporte de los EC para el tratamiento de las cicatrices queloides, y suponer que la heterogeneidad en los resultados obtenidos y la dificultad para realizar metaanálisis es producto de sus deficiencias metodológicas. A partir de este análisis, se proponen lineamientos para el diseño de EC sobre el tratamiento de las cicatrices queloides que puedan orientar al investigador clínico en su labor cotidiana. Finalmente, ante las nuevas terapias genéticas y epigenéticas para las cicatrices queloides, es necesario realizar EC con buena calidad metodológica para determinar si dichos tratamientos son eficaces desde el punto de vista clínico.

\section{Conflicto de intereses}

Los autores declaran que no existe conflicto de intereses.

\section{Responsabilidades éticas}

Protección de personas y animales. Los autores declaran que para esta investigación no se han realizado experimentos en seres humanos ni en animales.

Confidencialidad de los datos. Los autores declaran que han seguido los protocolos de su centro de trabajo sobre la publicación de datos de pacientes.

Derecho a la privacidad y consentimiento informado. Los autores declaran que en este artículo no aparecen datos de pacientes.

\section{Anexo}

Puede consultar el material adicional del anexo en https://doi.org/10.24875/CIRU.20001506.

\section{Bibliografía}

1. Sanclemente G. Evidencias en dermatología: ensayos clínicos, revisiones sistemáticas y guías de práctica clínica. Tesis doctoral. Departamento de Pediatría, Obstetricia, Ginecología y Medicina Preventiva, Universidad Autónoma de Barcelona, España; 2016.

2. Herranz P, Santos-Heredero X. Cicatrices. Guía de valoración y tratamiento. Meda Pharma. Publicidad Just in Time S.L., España; 2012.

3. Ogawa R. Keloid and hypertrophic scars are the result of chronic inflammation in the reticular dermis. Int J Mol Sci. 2017;18. pii: E606.

4. Ren Y, Zhou X, Wei Z, Lin W, Fan B, Feng S. Efficacy and safety of triamcinolone acetonide alone and in combination with 5-fluorouracil for treating hypertrophic scars and keloids: a systematic review and meta-analysis. Int Wound J. 2017;14:480-7.

5. Wong T-S, Li JZ-H, Chen S, Chan JY-W, Gao W. The efficacy of triamcinolone acetonide in keloid treatment: a systematic review and meta-analysis. Front Med. 2016;3:71.

6. Bi M, Sun P, Li D, Dong Z, Chen Z. Intralesional injection of botulinum toxin type A compared with intralesional injection of corticosteroid for the treatment of hypertrophic scar and keloid: a systematic review and meta-analysis. Med Sci Monit. 2019;25:2950-8.

7. Moraga J, Cartes-Velásquez R. Pautas de chequeo, parte I: Consort y Trend. Rev Chil Cir. 2015;67:225-32.

8. Morales-Sánchez MA, Peralta-Pedrero ML, Jurado-Santa Cruz F. Core outcomes of clinical trials for the treatment of keloid scars. Citado 08 abril 2019. Disponible en: https://www.crd.york.ac.uk/prospero/display_ record.php?ID=CRD42018107933

9. Moher D, Hopewell S, Schulz KF, Montori V, Gotzsche PC, Devereaux PJ, et al. CONSORT 2010 explanation and elaboration: updated guidelines for reporting parallel group randomized trials. BMJ. 2010;340:c869.

10. Higgins J, Green S. Cochrane Handbook for systematic reviews of interventions. Version 5.1.0, 2011. Disponible en: https://handbook-5-1.cochrane.org/

11. Vercelli S, Ferriero G, Sartorio F, Cisari C, Bravani E. Clinimetric properties and clinical utility in rehabilitation of postsurgical scar rating scales: a systematic review. Int J Rehabil Res. 2015;38:279-86.

12. Mundy LR, Miller HC, Klassen AF, Cano SJ, Pusic AL. Patient-reported outcome instruments for surgical and traumatic scars: a systematic review of their development, content, and psychometric validation. Aesthetic Plast Surg. 2016;40:792-800.

13. Tan A, Glass DA $2^{\text {nd }}$. Patient-reported outcomes for keloids: a systematic review. G Ital Dermatol Venereol. 2019;154:148-65.

14. Slemp AE, Kirschner RE. Keloids and scars: a review of keloids and scars, their pathogenesis, risk factors, and management. Curr Opin Pediatr. 2006;18:396-402.

15. Schmitt J, Apfelbacher C, Spuls PI, Thomas KS, Simpson EL, Furue M, et al. The harmonizing outcome measures for eczema (HOME) roadmap: a methodological framework to develop core sets of outcome measurements in dermatology. J Invest Dermatol. 2015;135:24-30. 


\begin{tabular}{|c|c|c|}
\hline Autor & Año & Referencia bibliográfica \\
\hline Aggarwal A & 2018 & $\begin{array}{l}\text { Aggarwal A, Ravikumar BC, Vinay KN, Raghukumar S, Yashovardhana } \\
\text { DP. A comparative study of various modalities in the treatment of keloids. Int } J \\
\text { Dermatol. 2018;57:1192-200. }\end{array}$ \\
\hline Ahuja RB & 2013 & $\begin{array}{l}\text { Ahuja RB, Chatterjee P. Comparative efficacy of intralesional verapamil } \\
\text { hydrochloride and triamcinolone acetonide in hypertrophic scars and } \\
\text { keloids. Burns. 2014;40:583-8. }\end{array}$ \\
\hline $\begin{array}{l}\text { Al-Mohamady } \\
\text { Ael-S }\end{array}$ & 2016 & $\begin{array}{l}\text { Al-Mohamady Ael-S, Ibrahim SM, Muhammad MM. Pulsed dye laser versus } \\
\text { long-pulsed Nd:YAG laser in the treatment of hypertrophic scars and keloid: A } \\
\text { comparative randomized split-scar trial. J Cosmet Laser Ther. 2016;18:208-12. }\end{array}$ \\
\hline Alster TS & 1995 & $\begin{array}{l}\text { Alster TS, Williams CM. Treatment of keloid sternotomy scars with } 585 \mathrm{~nm} \\
\text { flashlamp-pumped pulsed-dye laser. Lancet. 1995;345:1198-200. }\end{array}$ \\
\hline Asilian A & 2006 & $\begin{array}{l}\text { Asilian A, Darougheh A, Shariati F. New combination of triamcinolone, 5- } \\
\text { fluorouracil, and pulsed-dye laser for treatment of keloid and hypertrophic scars. } \\
\text { Dermatol Surg. 2006;32:907-15. }\end{array}$ \\
\hline Azzam OA & 2016 & $\begin{array}{l}\text { Azzam OA, Bassiouny DA, El-Hawary MS, El Maadawi ZM, Sobhi RM, } \\
\text { El-Mesidy MS. Treatment of hypertrophic scars and keloids by fractional carbon } \\
\text { dioxide laser: a clinical, histological, and immunohistochemical study. Lasers } \\
\text { Med Sci. 2016;31:9-18. }\end{array}$ \\
\hline Behera B & 2016 & $\begin{array}{l}\text { Behera B, Kumari R, Thappa DM, Malathi M. Therapeutic efficacy of } \\
\text { intralesional steroid with carbon dioxide laser versus with cryotherapy in } \\
\text { treatment of keloids: a randomized controlled trial. Dermatol Surg. } \\
\text { 2016;42:1188-98. }\end{array}$ \\
\hline Berman B & 1999 & $\begin{array}{l}\text { Berman B, Flores F. Comparison of a silicone gel-filled cushion and silicon gel } \\
\text { sheeting for the treatment of hypertrophic or keloid scars. Dermatol Surg. } \\
\text { 1999;25:484-6. }\end{array}$ \\
\hline
\end{tabular}




\begin{tabular}{|c|c|c|}
\hline Bijlard E & 2013 & $\begin{array}{l}\text { Bijlard E, Timman R, Verduijn GM, Niessen FB, van Neck JW, Busschbach } \\
\text { JJ, et al. Intralesional cryotherapy versus excision and corticosteroids or } \\
\text { brachytherapy for keloid treatment: study protocol for a randomised controlled } \\
\text { trial. Trials. } 2013 ; 14: 439 \text {. }\end{array}$ \\
\hline Bisbal J & 2009 & $\begin{array}{l}\text { Bisbal J, Guix B, Coronel R. Tratamiento combinado de los queloides mediante } \\
\text { cirugía y braquiterapia. Cirugía Plástica Ibero-Latinoamericana. 2009;35:283-90. }\end{array}$ \\
\hline Broker BJ & 1996 & $\begin{array}{l}\text { Broker BJ, Rosen D, Amsberry J, Schmidt R, Sailor L, Pribitkin EA, et al. } \\
\text { Keloid excision and recurrence prophylaxis via intradermal interferon- } \\
\text { gamma injections: a pilot study. Laryngoscope. 1996;106(12 Pt 1):1497- } \\
501 \text {. }\end{array}$ \\
\hline D'Andrea F & 2002 & $\begin{array}{l}\text { D'Andrea F, Brongo S, Ferraro G, Baroni A. Prevention and treatment of } \\
\text { keloids with intralesional verapamil. Dermatology. 2002;204:60-2. }\end{array}$ \\
\hline Darougheh A & 2008 & $\begin{array}{l}\text { Darougheh A, Asilian A, Shariati F. Intralesional triamcinolone alone or in } \\
\text { combination with 5-fluorouracil for the treatment of keloid and hypertrophic } \\
\text { scars. Clin Exp Dermatol. 2009;34:219-23. }\end{array}$ \\
\hline Davison SP & 2006 & $\begin{array}{l}\text { Davison SP, Mess S, Kauffman LC, Al-Attar A. Ineffective treatment of } \\
\text { keloids with interferon alpha-2b. Plast Reconstr Surg. 2006;117:247-52. }\end{array}$ \\
\hline Emad M & 2010 & $\begin{array}{l}\text { Emad M, Omidvari S, Dastgheib L, Mortazavi A, Ghaem H. Surgical excision } \\
\text { and immediate postoperative radiotherapy versus cryotherapy and intralesional } \\
\text { steroids in the management of keloids: a prospective clinical trial. Med Princ } \\
\text { Pract. 2010;19:402-5. }\end{array}$ \\
\hline Ghonaim N & 2013 & $\begin{array}{l}\text { Ghonaim N. Comparative study of the } 80 \% \text { trichloroacetic acid multiple puncture } \\
\text { technique versus botulinum toxin type A in the treatment of keloid scars. } \\
\text { Egyptian Journal of Dermatology and Venereology. } 2013 ; 33: 22-7 \text {. }\end{array}$ \\
\hline Har-Shai Y & 2007 & $\begin{array}{l}\text { Har-Shai Y, Dujovni ER, Zouboulis CC. Effect of skin surface temperature } \\
\text { on skin pigmentation during contact and intralesional cryosurgery of } \\
\text { keloids. J Eur Acad Dermatol Venerol. 2007;21:191-8. }\end{array}$ \\
\hline
\end{tabular}




\begin{tabular}{|c|c|c|}
\hline Hatamipor E & 2010 & $\begin{array}{l}\text { Hatamipour E, Mehrabi S, Hatamipour M, Ghafarian Shirazi HR. Effects of } \\
\text { combined intralesional 5-fluorouracil and topical silicone in prevention of keloids: } \\
\text { a double blind randomized clinical trial study. Acta Med Iran. 2011;49:127-30. }\end{array}$ \\
\hline $\begin{array}{l}\text { Hedayatyanfard } \\
\mathrm{K}\end{array}$ & 2018 & $\begin{array}{l}\text { Hedayatyanfard K, Ziai SA, Niazi F, Habibi I, Habibi B, Moravvej H. } \\
\text { Losartan ointment relieves hypertrophic scars and keloid: a pilot study. Wound } \\
\text { Repair Regen. 2018;26:340-3. }\end{array}$ \\
\hline Hosnuter M & 2007 & $\begin{array}{l}\text { Hosnuter M, Payasli C, Isikdemir A, Tekerekoglu B. The effects of onion } \\
\text { extract on hypertrophic and keloid scars. J Wound Care. 2007;16:251-4. }\end{array}$ \\
\hline Khan MA & 2014 & $\begin{array}{l}\text { Khan MA, Bashir MM, Khan FA. Intralesional triamcinolone alone and in } \\
\text { combination with 5-fluorouracil for the treatment of keloid and hypertrophic } \\
\text { scars. J Pak Med Assoc. 2014;64:1003-7. }\end{array}$ \\
\hline Kill J & 1977 & $\begin{array}{l}\text { Kill J. Keloids treated with topical injections of triamcinolone acetonide } \\
\text { (kenalog). Immediate and long-term results. Scand J Plast Reconstr Surg. } \\
\text { 1977;11:169-72. }\end{array}$ \\
\hline Koc E & 2008 & $\begin{array}{l}\text { Koc E, Arca E, Surucu B, Kurumlu Z. An open, randomized, controlled, } \\
\text { comparative study of the combined effect of intralesional triamcinolone } \\
\text { acetonide and onion extract gel and intralesional triamcinolone acetonide } \\
\text { alone in the treatment of hypertrophic scars and keloids. Dermatol Surg. } \\
\text { 2008;34:1507-14. }\end{array}$ \\
\hline
\end{tabular}

Lawrence T 1991 Lawrence WT. In search of the optimal treatment of keloids: report of a series and a review of the literature. Ann Plast Surg. 1991;27:164-78.

Layton AM

1994 Layton AM, Yip J, Cunliffe WJ. A comparison of intralesional triamcinolone and cryosurgery in the treatment of acne keloids. Br J Dermatol. 1994;130:498501.

Lee JH

2008 Lee JH, Kim SE, Lee AY. Effects of interferon-alpha $2 b$ on keloid treatment with triamcinolone acetonide intralesional injection. Int J Dermatol. 2008; 47:183-6. 
Mandello S 2015 Carvalhaes SM, Petroianu A, Ferreira MAT, Barros VM de, Lopes RV. Assessment of the treatment of earlobe keloids with triamcinolone injections, surgical resection, and local pressure. Rev Col Bras Cir. 2015;42:9-13.

\begin{abstract}
Manuskiatti W 2002 Manuskiatti W, Fitzpatrick RE. Treatment response of keloidal and hypertrophic sternotomy scars: comparison among intralesional corticosteroid, 5fluorouracil, and 585-nm flashlamp-pumped pulsed-dye laser treatments. Arch Dermatol. 2002;138:1149-55.

Manuskiatti W 2007 Manuskiatti W, Wanitphakdeedecha R, Fitzpatrick RE. Effect of pulse width of a 595-nm flashlamp-pumped pulsed dye laser on the treatment response of keloidal and hypertrophic sternotomy scars. Dermatol Surg. 2007;33:152-61.

Mourad B 2016 Mourad B, Elfar N, Elsheikh S. Spray versus intralesional cryotherapy for keloids. J Dermatolog Treat. 2016;27:264-9.

Naeni FF 2006 Naeini FF, Najafian J, Ahmadpour K. Bleomycin tattooing as a promising therapeutic modality in large keloids and hypertrophic scars. Dermatol Surg. 2006;32:1023-9; discussion 1029-30.
\end{abstract}

Nor NM

2016 Nor NM, Ismail R, Jamil A, Shah SA, Imran FH. A randomized, single-blind trial of clobetasol propionate $0.05 \%$ cream under silicone dressing occlusion versus intra-lesional triamcinolone for treatment of keloid. Clin Drug Investig. 2017;37:295-301.

De Oliveira GV 2001 De Oliveira GV, Nunes TA, Magna LA, Cintra ML, Kitten GT, Zarpellon $\mathrm{S}$, et al. Silicone versus nonsilicone gel dressings: a controlled trial. Dermatol Surg. 2001;27:721-6.

Palmieri B 1995 Palmieri B, Gozzi G, Palmieri G. Vitamin E added silicone gel sheets for treatment of hypertrophic scars and keloids. Int J Dermatol. 1995;34:506-9.

Paquet $\mathrm{P}$

2001 Paquet P, Hermanns JF, Piérard GE. Effect of the $585 \mathrm{~nm}$ flashlamp-pumped pulsed dye laser for the treatment of keloids. Dermatol Surg. 2001;27:171-4. 


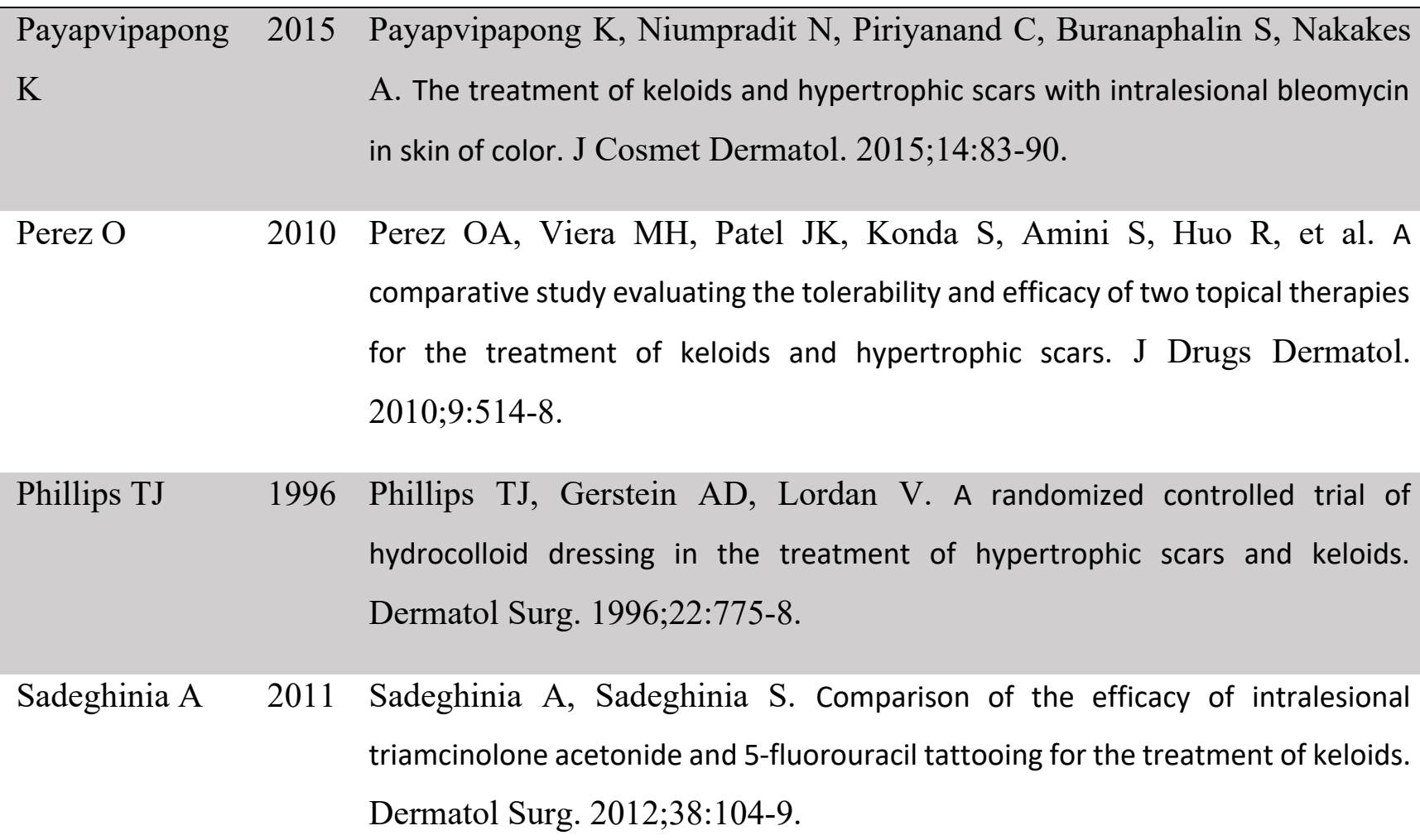

Perez O 2010 Perez OA, Viera MH, Patel JK, Konda S, Amini S, Huo R, et al. A comparative study evaluating the tolerability and efficacy of two topical therapies for the treatment of keloids and hypertrophic scars. J Drugs Dermatol. 2010;9:514-8.

Phillips TJ 1996 Phillips TJ, Gerstein AD, Lordan V. A randomized controlled trial of hydrocolloid dressing in the treatment of hypertrophic scars and keloids. Dermatol Surg. 1996;22:775-8.

Sadeghinia A 2011 Sadeghinia A, Sadeghinia S. Comparison of the efficacy of intralesional triamcinolone acetonide and 5-fluorouracil tattooing for the treatment of keloids. Dermatol Surg. 2012;38:104-9.
Santos JMP 2015 Santos JMP dos, Souza C de, Vasconcelos AC de, Nunes TA. Effect of triamcinolone in keloids morphological changes and cell apoptosis. Rev Col Bras Cir. 2015;42:171-4.
Sawada Y
1992 Sawada Y, Sone K. Hydration and occlusion treatment for hypertrophic scars and keloids. Br J Plast Surg. 1992;45:599-603.
Sawada Y 1990 Sawada Y, Sone K. Treatment of scars and keloids with a cream containing silicone oil. Br J Plast Surg. 1990;43:683-8.
Sclafani AP 1996 Sclafani AP, Gordon L, Chadha M, Romo T 3rd. Prevention of earlobe keloid recurrence with postoperative corticosteroid injections versus radiation therapy: a randomized, prospective study and review of the literature. Dermatol Surg. 1996;22:569-74.
Shaarawy E 2015 Shaarawy E, Hegazy RA, Abdel Hay RM. Intralesional botulinum toxin type A equally effective and better tolerated than intralesional steroid in the treatment of keloids: a randomized controlled trial. J Cosmet Dermatol. 2015;14:161-6.




\begin{tabular}{|c|c|c|}
\hline Shanthi FXM & 2008 & $\begin{array}{l}\text { Shanthi FXM, Ernest K, Dhanraj P. Comparison of intralesional verapamil with } \\
\text { intralesional triamcinolone in the treatment of hypertrophic scars and keloids. } \\
\text { Indian J Dermatol Venereol Leprol. 2008;74:343-8. }\end{array}$ \\
\hline Sharma S & 2007 & $\begin{array}{l}\text { Sharma S, Bhanot A, Kaur A, Dewan SP. Role of liquid nitrogen alone } \\
\text { compared with combination of liquid nitrogen and intralesional triamcinolone } \\
\text { acetonide in treatment of small keloids. J Cosmet Dermatol. 2007;6:258-61. }\end{array}$ \\
\hline Tan $\mathrm{C}$ & 2018 & $\begin{array}{l}\text { Tan C, Yeo Chen Long D, Cao T, Tan Wei Ding V, Srivastava R, Yow AP, } \\
\text { et al. Drug-free microneedles in the treatment of keloids: a single-blinded } \\
\text { intraindividual controlled clinical trial. Br J Dermatol. 2018;179:1418-9. }\end{array}$ \\
\hline Uppal RS & 2001 & $\begin{array}{l}\text { Uppal RS, Khan U, Kakar S, Talas G, Chapman P, McGrouther AD. The } \\
\text { effects of a single dose of 5-fluorouracil on keloid scars: a clinical trial of timed } \\
\text { wound irrigation after extralesional excision. Plast Reconstr Surg. } \\
\text { 2001;108:1218-24. }\end{array}$ \\
\hline Wananukul S & 2013 & $\begin{array}{l}\text { Wananukul S, Chatpreodprai S, Peongsujarit D, Lertsapcharoen P. A } \\
\text { prospective placebo-controlled study on the efficacy of onion extract in silicone } \\
\text { derivative gel for the prevention of hypertrophic scar and keloid in median } \\
\text { sternotomy wound in pediatric patients. J Med Assoc Thai. 2013;96:1428-33. }\end{array}$ \\
\hline Wang CJ & 2018 & $\begin{array}{l}\text { Wang CJ, Ko JY, Chou WY, Cheng JH, Kuo YR. Extracorporeal shockwave } \\
\text { therapy for treatment of keloid scars. Wound Repair Regen. 2018;26:69-76. }\end{array}$ \\
\hline Yosipovitch G & 2016 & $\begin{array}{l}\text { Yosipovitch G, Widijanti Sugeng M, Goon A, Chan YH, Goh CL. A } \\
\text { comparison of the combined effect of cryotherapy and corticosteroid injections } \\
\text { versus corticosteroids and cryotherapy alone on keloids: a controlled study. J } \\
\text { Dermatolog Treat. 2001;12:87-90. }\end{array}$ \\
\hline Zhang M & 2018 & $\begin{array}{l}\text { Zhang M, Liu S, Guan E, Liu H, Dong X, Hao Y, et al. Hyperbaric oxygen } \\
\text { therapy can ameliorate the EMT phenomenon in keloid tissue. Medicine (Balt). } \\
\text { 2018;97:e11529. }\end{array}$ \\
\hline
\end{tabular}

